\title{
The predictive value of the preoperative fibrinogen-albumin ratio on the postoperative prognosis of renal cell carcinoma
}

\author{
Jun Liu ${ }^{1,2,3,4,5 \#}$, Ying Gan ${ }^{1,2,3 \#}$, Haifeng Song ${ }^{1,2,3}$, Kun Zhu ${ }^{1,2,3}$, Qian Zhang ${ }^{1,2,3}$ \\ ${ }^{1}$ Department of Urology, Peking University First Hospital, Beijing 100034, China; ${ }^{2}$ Institute of Urology, Peking University, Beijing 100034, China; \\ ${ }^{3}$ National Research Center for Genitourinary Oncology, Beijing 100034, China; ${ }^{4}$ Peking University Applied Lithotripsy Institute, Peking University, \\ Beijing 100044, China; ${ }^{5}$ Urology and lithotripsy center, Peking University People's Hospital, Beijing 100044, China \\ Contributions: (I) Conception and design: Q Zhang; (II) Administrative support: Y Gan; (III) Provision of study materials or patients: J Liu, Y Gan; \\ (IV) Collection and assembly of data: H Song, K Zhu; (V) Data analysis and interpretation: J Liu; (VI) Manuscript writing: All authors; (VII) Final \\ approval of manuscript: All authors. \\ \#These authors contributed equally to this work. \\ Correspondence to: Qian Zhang. Department of Urology, Peking University First Hospital, 8 Xishiku Street, Xicheng District, Beijing 100034, China. \\ Email: zhangqianbjmu@126.com.
}

\begin{abstract}
Background: Urologists urgently need a simple, effective, accurate clinical biomarker to identify renal cell carcinoma (RCC) patients with poor prognosis and those with a high risk of recurrence as early as possible. Therefore, we investigated the prognostic value of the preoperative fibrinogen-albumin ratio (FAR) in patients with RCC.

Methods: We retrospectively analyzed data from 279 cases of renal cancer admitted to the First Hospital of Peking University from 2010 to 2012. The best cutoff value of the FAR was obtained using receiver operating characteristic (ROC) curve analysis, and patients were divided into high- and low-FAR groups. The correlation between the preoperative FAR and clinicopathological features was analyzed by $\chi^{2}$ test. Log-rank test and Cox proportional hazard regression model were used to evaluate the predictive value of clinicopathological parameters for overall survival (OS).

Results: The best cutoff value for the FAR was 0.116. A FAR $>0.116$ was associated with higher Fuhrman grade $(\mathrm{P}<0.0001)$ and later pathological $\mathrm{T}$ stage $(\mathrm{P}<0.0001)$. Patients with a high $\mathrm{FAR}(>0.116)$ had worse OS [hazard ratio (HR) 10.497, 95\% confidence interval (CI): 3.263-33.766, $\mathrm{P}<0.0001$ ]. In multivariate analysis, the FAR was an independent risk factor for OS (HR 5.047, 95\% CI: 2.109-12.076, P=0.003). Moreover, in Fuhrman grade I-II patients, the FAR could distinguish patients with worse prognosis $(\mathrm{P}<0.0001)$.

Conclusions: The preoperative FAR is an independent prognostic factor of OS in renal cancer patients. A FAR $>0.116$ was significantly related to decreased survival in renal cancer patients.
\end{abstract}

Keywords: Albumins; biomarkers; fibrinogen; renal cell carcinoma (RCC); prognosis

Submitted Dec 16, 2019. Accepted for publication May 13, 2020.

doi: $10.21037 /$ tau-19-873

View this article at: http://dx.doi.org/10.21037/tau-19-873

\section{Introduction}

Renal cell carcinoma (RCC) is one of the most common malignant tumors of the urinary system, and it is the 6th most common cancer in men and the 10th in women. RCC accounts for $5 \%$ of all tumors in men and $3 \%$ of all tumors in women (1). Although most patients are diagnosed at early stages, a considerable proportion of renal cancer is only diagnosed in the local advanced stage, and $17 \%$ of patients have distant metastasis at the time of diagnosis (2). In recent years, due to the great progress of anti-angiogenic drugs and immunotherapy $(3,4)$, many patients with advanced renal cancer have achieved good therapeutic effect, which has changed the natural course of renal cancer. Therefore, 
urologists urgently need a simple, effective, accurate clinical biomarker to screen out patients with poor prognosis and identify patients with a high risk of recurrence and poor prognosis as early as possible. Identifying such a biomarker can also provide accurate clinical decision-making and active follow-up monitoring.

Fibrinogen is a kind of glycoprotein that is mainly produced in the liver and secreted into the serum. A number of recent epidemiological studies have shown that fibrinogen not only participates in coagulation but also plays a role in the occurrence and development of tumors. Previous studies have shown that an increase in preoperative fibrinogen is associated with the progression and poor prognosis of malignant tumors $(5,6)$. In addition, hypoproteinemia has been found to be associated with poor prognosis in a variety of cancers, including lung cancer, gastric cancer, and colon cancer (7-9). The fibrinogenalbumin ratio (FAR), as well as fibrinogen and albumin as single factors, are related to poor prognosis in esophageal cancer, liver cancer, and other tumors $(10,11)$. Therefore, we assume that the FAR has potential value in evaluating the prognosis of patients with nonmetastatic renal cancer. The purpose of this study was to evaluate the prognostic value of the preoperative FAR in patients with renal cancer after resection and to compare it with established systemic inflammatory immune biomarkers, including the neutrophil-lymphocyte ratio (NLR), prognostic nutrition index (PNI), and controlling nutritional status (CONUT) score.

We present the following article in accordance with the STROBE reporting checklist (available at http://dx.doi. org/10.21037/tau-19-873).

\section{Methods}

\section{Patients}

From 2010 to 2012, 279 patients with renal cancer who underwent radical nephrectomy or partial nephrectomy were collected from the Urology Department of Peking University First Hospital. All cases were confirmed by postoperative pathology. All patients had no history of other types of malignant tumor, no lymph node metastasis or distant metastasis, no thrombotic disease, and no history of anticoagulation treatment. The clinicopathological data were obtained from the patient's electronic medical record, and the pathological and clinical data of all cases were complete. During the follow-up of this study, the patients or their relatives were informed about the content of this study in detail and got their oral consent. The study was approved by the central ethics committee of our hospital.

\section{Clinical data and peripheral blood biomarkers}

In all patients included in the study, serum fibrinogen, albumin, neutrophil count, lymphocyte count, and serum total cholesterol level were measured within 1 week before operation. Other factors were calculated as follows: FAR = total fibrinogen/total albumin, NLR = absolute neutrophil count/absolute lymphocyte count, and PNI = albumin $(\mathrm{g} / \mathrm{dL})+5 \times$ absolute lymphocyte count $\left(10^{9} / \mathrm{L}\right)$. The CONUT score was calculated as described previously $(12,13)$.

\section{Follow-up}

The patients were followed up every 3 months in the first 2 years and every 6 months thereafter. Patients were followed up by blood examination, biochemical tests, chest radiography, and abdominal ultrasound/abdominal CT. The follow-up methods included telephone follow-up and outpatient follow-up.

\section{Statistical analysis}

The end point of this study was overall survival (OS). OS was defined as the time from the operation to death from any cause.

SPSS 24.0 software was used to analyze the data. The best cutoff value for the FAR was calculated by receiver operating characteristic (ROC) curve analysis. Patients were divided into two groups based on the best cutoff value (high-FAR and low-FAR), and the clinical and pathological characteristics between the two groups were compared, including age, gender, hypertension, diabetes, Fuhrman grade, tumor classification, pathological T stage, histological subtype, and operation method. $\chi^{2}$ test was used for the comparison of count data. The Kaplan-Meier method was used to calculate the survival rate and draw survival curves. The log-rank test was used to compare survival differences between groups. Cox hazards regression model was used to analyze the prognostic factors and determine the independent predictors of OS. A bilateral 


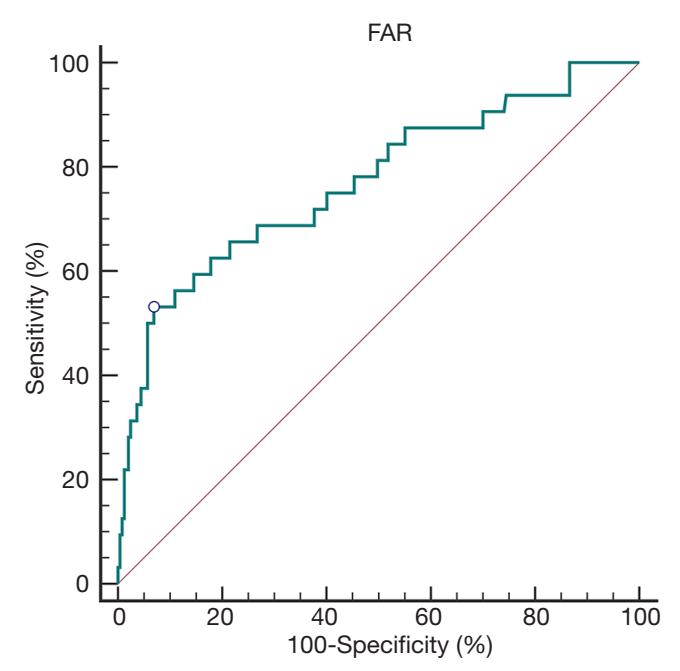

Figure 1 Determination of the cut-off value for the FAR by ROC curve analysis. FAR, fibrinogen-albumin ratio; ROC, receiver operating characteristic.

$\mathrm{P}<0.05$ was considered statistically significant.

\section{Results}

\section{Determination of the optimal cutoff value of the FAR}

The best cutoff value for the preoperative FAR was determined by ROC curve analysis (Figure 1). At a FAR of 0.116 , the Youden index of the FAR was highest, at 0.462 ( sensitivity $=53.13 \%$, specificity $=93.12 \%$ ); therefore, 0.116 was selected as the cutoff value of the FAR (AUC: 0.769, 95\% CI: 0.715-0.817, $\mathrm{P}<0.0001)$.

\section{The relationship between the preoperative FAR and clinicopathological factors of RCC}

Of the 279 patients included in the study, 195 were men and 84 were women. The median follow-up time was 65 months (range, 2-75 months). Table 1 summarizes the detailed baseline characteristics of the selected patients. According to the best cutoff value of the FAR as determined by ROC curve analysis, the patients were divided into two groups. There were 35 patients in the high-FAR $(>0.116)$ group and 244 patients in the low-FAR $(\leq 0.116)$ group. A FAR $>0.116$ was significantly correlated with high Fuhrman grade $(\mathrm{P}<0.0001)$ and late pathological $\mathrm{T}$ stage $(\mathrm{P}<0.0001)$ (Table 1$)$. There was no significant correlation between FAR and age, gender, diabetes, hypertension, tumor histology, or operation method.

\section{The relationship between clinicopathological features and prognosis of RCC}

Kaplan-Meier survival analysis indicated that a FAR $>0.116$ was associated with worse OS (Figure 2). In order to further determine the predictors of postoperative OS, we evaluated the FAR and other clinicopathological parameters by Cox proportional hazards regression analysis. In univariate regression analysis, higher Fuhrman grade $(\mathrm{P}<0.0001)$, higher pathological $\mathrm{T}$ stage $(\mathrm{P}<0.0001)$, laparoscopy $(\mathrm{P}=0.021)$, and a FAR $>0.116(\mathrm{P}<0.0001)$ were associated with shorter OS. Multiple regression analysis indicated that Fuhrman grade and the FAR were independent prognostic factors of OS (Table 2).

\section{Comparison of the AUCs for the FAR, NLR, PNI, and CONUT score}

We use the AUCs to estimate the accuracy of the FAR for 5 -year OS prediction. The AUC scores of the NLR, PNI, and CONUT score were 0.751 (95\% CI: 0.696-0.801), 0.697 (95\% CI: $0.639-0.750$ ), and 0.728 (95\% CI: $0.672-$ $0.780)$, respectively. The AUC of the FAR was 0.769 (95\% CI: 0.715-0.817), which was higher than those of the NLR, PNI, and CONUT score (Figure 3). However, there was no statistical difference in the AUCs, indicating that the FAR had the same accuracy in predicting the 5-year OS as the NLR, PNI, and CONUT score (Table 3).

\section{Prognostic significance of the FAR based on Fubrman grade}

We further studied the prognostic significance of the FAR in patients with Fuhrman I-II RCC and found that the FAR was significantly correlated with OS $(\mathrm{P}<0.0001)$ (Figure 4A). However, in Fuhrman III-IV RCC patients, the FAR did not distinguish patients with worse OS $(\mathrm{P}=0.059)$ (Figure $4 B)$. Further, in Fuhrman I-II RCC, the patients with FAR $\leq 0.16$, have a better OS $(\mathrm{P}<0.0001)$, the FAR was a prognostic factor for OS in Fuhrman grade I-II renal cancer patients.

\section{Discussion}

The FAR reflects the ratio of fibrinogen to albumin, and it has been shown to have good prognostic value in esophageal cancer (10) and hepatocellular carcinoma (HCC) (11). Inspired by this, we explored the prognostic 
Table 1 Comparison of clinicopathological data of different FAR groups

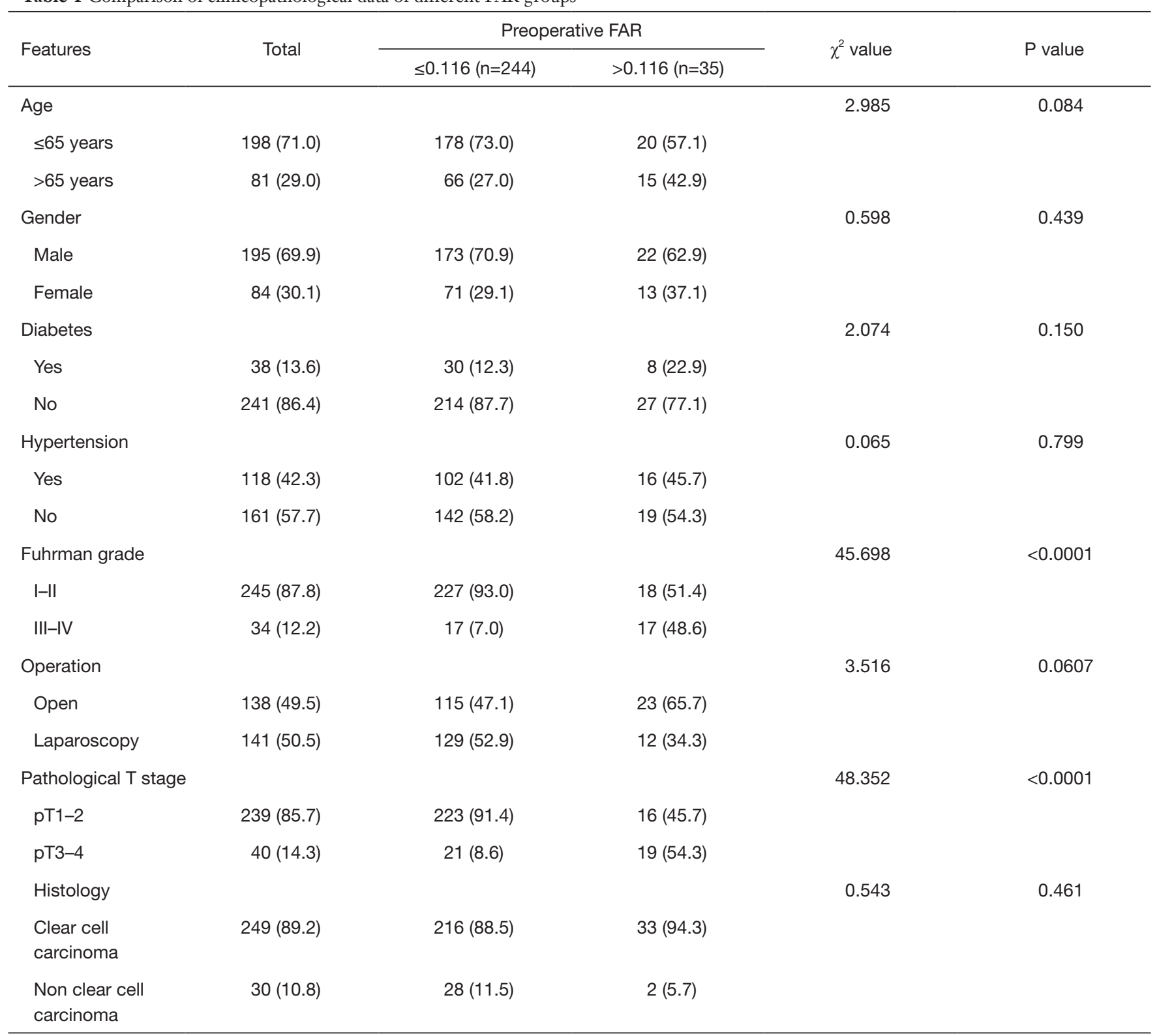

Data are presented as $n$ (\%). FAR, fibrinogen-albumin ratio.

value of the FAR after radical nephrectomy. To our knowledge, this is the first study to focus on the impact of the FAR on the prognosis of RCC.

In this study, we explored the FAR as a predictor of renal cancer prognosis. We divided the patients into two groups based on the best cutoff value for the FAR as determined by ROC curve analysis. There were 35 patients in the high-FAR $(>0.116)$ group and 244 patients in the low-FAR $(\leq 0.116)$ group. A high FAR was significantly correlated with high tumor grade $(\mathrm{P}<0.0001)$ and late pathological $\mathrm{T}$ stage $(\mathrm{P}<0.0001)$ (Table 1). In addition, multivariate Cox regression analysis showed that Fuhrman grade and the FAR were independent prognostic factors of OS. Both fibrinogen and albumin are routinely measured in the clinic. Therefore, using the FAR to assess prognosis would be cheap, convenient, and repeatable.

The nuclear grade, pathological stage, and pathological type of tumor have commonly been used as indexes to 


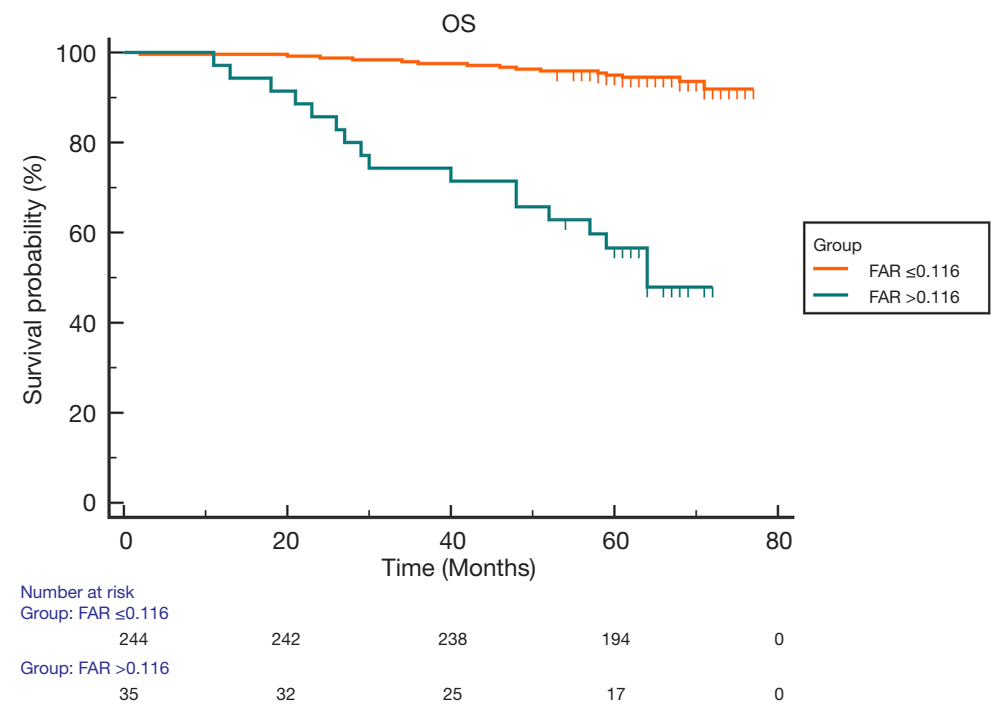

Figure 2 Kaplan-Meier survival curves for RCC patients. The OS of patients with a FAR $>0.116$ was significantly shorter than that of those with a FAR $\leq 0.116$ ( $\mathrm{P}<0.0001$, log-rank test). RCC, renal cell carcinoma; OS, overall survival; FAR, fibrinogen-albumin ratio.

Table 2 Univariate and multivariate analyses of clinicopathological variables to predict OS in patients with RCC

\begin{tabular}{|c|c|c|c|c|}
\hline \multirow{3}{*}{ Variables } & \multicolumn{4}{|c|}{ Overall survival } \\
\hline & \multicolumn{2}{|c|}{ Univariable } & \multicolumn{2}{|c|}{ Multivariable } \\
\hline & $\mathrm{HR}(95 \% \mathrm{Cl})$ & $P$ value & $\mathrm{HR}(95 \% \mathrm{Cl})$ & $P$ value \\
\hline Age (>65 vs. $\leq 65$ years) & $2.029(1.009-4.081)$ & 0.047 & - & - \\
\hline Gender (male vs. female) & $0.899(0.416-1.942)$ & 0.786 & - & - \\
\hline Diabetes (yes vs. no) & $2.203(0.989-4.904)$ & 0.053 & - & - \\
\hline Fuhrman grade (I-II vs. III-IV) & $9.035(4.512-18.092)$ & $<0.0001$ & $2.214(1.031-4.755)$ & 0.042 \\
\hline Histology (non-clear cell vs. clear cell) & $1.211(0.425-3.455)$ & 0.721 & - & - \\
\hline Pathological T stage (T3-T4 vs. T1-T2) & $6.491(3.236-13.019)$ & $<0.0001$ & $1.401(0.525-3.738)$ & 0.502 \\
\hline
\end{tabular}

OS, overall survival; RCC, renal cell carcinoma; HR, hazard ratio; 95\% Cl, 95\% confidence interval; FAR, fibrinogen-albumin ratio.

evaluate the prognosis of patients with RCC (14). However, these indexes are obtained by postoperative pathology, and more accurately identifying patients with poor prognosis before operation and carrying out tumor risk stratification is vital to determine treatment plans and guide postoperative follow-up. Therefore, finding biomarkers that can accurately predict tumor prognosis is of great interest. As early as the 19th century, Professor Rudolf Virchow first showed that there might be a connection between inflammation and cancer by observing the leukocytes in tumors. It is now generally believed that inflammation affects every step of tumor development, from tumorigenesis to metastasis (15).

Fibrinogen is not only an important factor to maintain normal coagulation function but also an acute phase reactive protein that can reflect the systemic inflammatory response. The systemic inflammatory response and coagulation are closely related to tumor development (16). Many recent studies have shown that preoperative peripheral blood 
fibrinogen levels are related to survival in a variety of malignant tumors. Further, higher plasma fibrinogen levels are related to poor pathological characteristics and can predict survival in lung cancer, breast cancer, ovarian cancer, colon cancer, prostate cancer, and other tumors (17-21). This may be related to the direct involvement of fibrinogen in the interaction between vascular endothelial growth factor, transforming growth factor- $\beta$, platelet-derived growth factor, and fibroblast growth factor to regulate angiogenesis. Fibrinogen also plays a key role in cell proliferation, angiogenesis, and hematogenous metastasis of tumor cells (22-24).

At present, serum albumin levels are the most direct laboratory index to evaluate the nutritional status of patients.

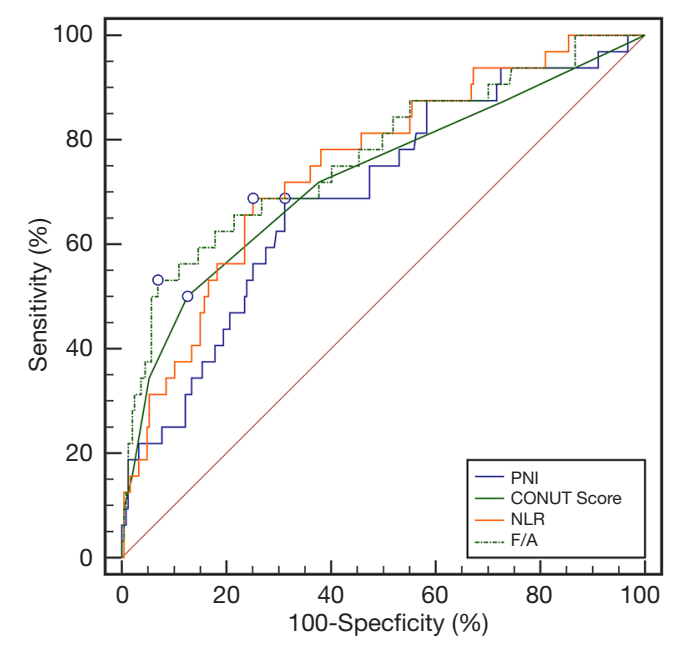

Figure 3 Comparison of the areas under the curves for the FAR, NLR, PNI, and CONUT score. The discrimination abilities of the FAR, NLR, PNI, and CONUT score for OS were compared. FAR, fibrinogen-albumin ratio; NLR, neutrophil-lymphocyte ratio; PNI, prognostic nutrition index; CONUT, controlling nutritional status; OS, overall survival.
Studies have shown that the inflammatory response and tumor state also affect serum albumin concentration. Low albumin levels may weaken the immune system, increase the chance of infection, and further accelerate the development of malignant tumors (25). Albumin has been reported to be of prognostic value in a variety of human malignant tumors: the lower the serum albumin level, the worse the prognosis of cancer patients, including in head and neck cancer (26), non-small cell lung cancer (27), ovarian cancer (28), and adenocarcinoma of the gastric cardia (29). In addition, serum albumin is one of the components of the Child-Pugh classification system reflecting liver function. Studies have shown that hypoproteinemia is an independent prognostic factor for poor prognosis in patients with liver cancer (30).

Other preoperative blood indexes based on inflammation and nutritional metabolism, such as the NLR, PNI, and CONUT score, have recently been reported as useful prognostic indexes of renal cancer $(12,13,31,32)$. We compared the FAR to these common indexes in predicting the 5-year OS after surgery and found that the FAR had the same or better accuracy in predicting the 5-year OS.

Clinically, Fuhrman classification is considered to be an important factor influencing the prognosis of renal cancer patients after surgery (14). A higher Fuhrman grade indicates worse prognosis and shorter OS. However, the prognosis is still poor in some Fuhrman grade I-II patients. Therefore, it is of great clinical significance to find a predictor to distinguish high-risk patients in this subgroup. In our study, Fuhrman grade was an independent prognostic factor in patients with RCC, and patients with Fuhrman grade III-IV had worse OS $(\mathrm{P}<0.0001)$. After stratifying the patient cohort according to the Fuhrman grade, we found that in Fuhrman III-IV RCC patients, the FAR does not distinguish patients with worse OS $(\mathrm{P}=0.059)$, although this may be due to the small sample size in the subgroup. However, in Fuhrman grade I-II RCC patients,

Table 3 Comparison of the areas under the curves for the FAR, NLR, PNI, and CONUT score

\begin{tabular}{llll}
\hline Markers & AUC & HR (95\% Cl) & P value \\
\hline FAR (dichotomized) & 0.769 & $0.715-0.817$ & 0.140 \\
NLR (dichotomized) & 0.751 & $0.696-0.801$ & 0.534 \\
PNI (dichotomized) & 0.697 & $0.639-0.750$ & 0.271 \\
CONUT score (dichotomized) & 0.728 & $0.672-0.780$ & 0.538 \\
\hline
\end{tabular}

AUC, area under the curve; HR, hazard ratio; 95\% Cl, 95\% confidence interval; FAR, fibrinogen-albumin ratio; NLR, neutrophil-lymphocyte ratio; PNI, prognostic nutrition index; CONUT, controlling nutritional status. 

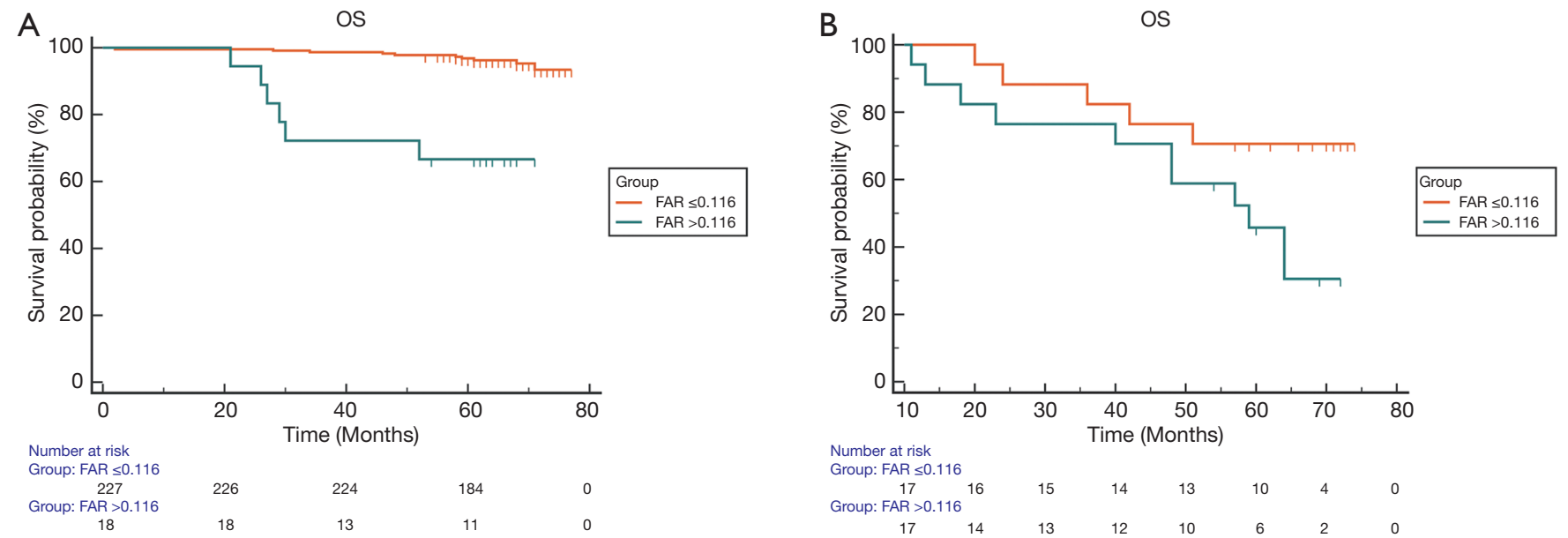

Figure 4 Kaplan-Meier survival curves of RCC patients with Fuhrman grade I-II (A, n=245) and Fuhrman grade III-IV (B, n=34). A FAR $>0.116$ was significantly correlated with a shorter OS in patients with Fuhrman grade I-II. RCC, renal cell carcinoma; OS, overall survival; FAR, fibrinogen-albumin ratio.

the prognostic significance of the FAR was very strong. Therefore, patients with Fuhrman grade I-II RCC with a high FAR may need to be followed up more closely as soon as possible because they are more likely to have worse OS. If early prediction and timely intervention can be achieved, these patients may achieve better results.

Other predictive biomarkers, such as Glasgow predictive score (GPS), platform to lymphocyte ratio (PLR), C-reactive protein to albumin ratio, in different studies, the results have a certain predictive effect on the prognosis of renal cancer (33-35). In this study, we found that FAR is a new and simple prognostic factor for renal cancer patients after surgery. However, there are some unavoidable limitations in this study. First, this is a single-center retrospective study. The results may be affected by selection bias. Whether our findings are applicable to other centers needs to be verified. Second, many other factors affect the FAR, such as acute and chronic infection and chronic liver disease; this may affect the accuracy of prognosis prediction based on this ratio. In addition, because the study population collected in this study was relatively early, many patients received different treatment measures due to tumor recurrence during follow-up, which affected OS.

In conclusion, despite these limitations, our study is the first to prove that the preoperative FAR is a new and simple prognostic factor for renal cancer patients after surgery, especially for patients with low Fuhrman grade. It is a simple and sensitive method to evaluate the prognosis of renal cancer patients that does not increase the economic burden or physical pain of patients and a promising biomarker.

\section{Acknowledgments}

Funding: This work was supported by the National Natural Science Foundation of China (Grant No. 81572510 and 81872088 to QZ) and the Scientific Research Seed Fund of Peking University First Hospital (Grant No. 2018 SF026 to YG).

\section{Footnote}

Reporting Checklist: The authors have completed the STROBE reporting checklist. Available at http://dx. doi. org/10. 21037/tau-19-873

Data Sharing Statement: Available at http://dx. doi. org/10.21037/tau-19-873

Conflicts of Interest: All authors have completed the ICMJE uniform disclosure form (available at http://dx. doi. org/10.21037/tau-19-873). QZ serves as an unpaid editorial board member of Translational Andrology and Urology from May 2019 to Apr 2021. The other authors have no conflicts of interest to declare.

Ethical Statement: The authors are accountable for all aspects of the work in ensuring that questions related to the accuracy or integrity of any part of the work are 
appropriately investigated and resolved. The study was approved by the Central Ethics Committee of our hospital (No. 2018-386).

Open Access Statement: This is an Open Access article distributed in accordance with the Creative Commons Attribution-NonCommercial-NoDerivs 4.0 International License (CC BY-NC-ND 4.0), which permits the noncommercial replication and distribution of the article with the strict proviso that no changes or edits are made and the original work is properly cited (including links to both the formal publication through the relevant DOI and the license). See: https://creativecommons.org/licenses/by-nc-nd/4.0/.

\section{References}

1. Siegel RL, Miller KD, Jemal A. Cancer statistics, 2018. CA Cancer J Clin 2018;68:7-30.

2. Capitanio U, Montorsi F. Renal cancer. Lancet 2016;387:894-906.

3. Lee CH, Motzer RJ. Kidney cancer in 2016: The evolution of anti-angiogenic therapy for kidney cancer. Nat Rev Nephrol 2017;13:69-70.

4. Thoma C. Kidney cancer: Combining targeted and immunotherapy. Nat Rev Urol 2018;15:263.

5. Ghanim B, Hoda MA, Klikovits T, et al. Circulating fibrinogen is a prognostic and predictive biomarker in malignant pleural mesothelioma. Br J Cancer 2014;110:984-90.

6. Son HJ, Park JW, Chang HJ, et al. Preoperative plasma hyperfibrinogenemia is predictive of poor prognosis in patients with nonmetastatic colon cancer. Ann Surg Oncol 2013;20:2908-13.

7. Gupta D, Lis CG. Pretreatment serum albumin as a predictor of cancer survival: a systematic review of the epidemiological literature. Nutr J 2010;9:69.

8. Wang Y, Li S, Hu X, et al. The prognostic value of serum albumin-globulin ratio in early-stage non-small cell lung cancer: a retrospective study. Cancer Manag Res 2019;11:3545-54.

9. Ouyang X, Dang Y, Zhang F, et al. Low Serum Albumin Correlates with Poor Survival in Gastric Cancer Patients. Clin Lab 2018;64:239-45.

10. Matsuda S, Takeuchi H, Kawakubo H, et al. Cumulative prognostic scores based on plasma fibrinogen and serum albumin levels in esophageal cancer patients treated with transthoracic esophagectomy: comparison with the Glasgow prognostic score. Ann Surg Oncol
2015;22:302-10.

11. Xu Q, Yan Y, Gu S, et al. A Novel InflammationBased Prognostic Score: The Fibrinogen/Albumin Ratio Predicts Prognoses of Patients after Curative Resection for Hepatocellular Carcinoma. J Immunol Res 2018;2018:4925498.

12. Elghiaty A, Kim J, Jang WS, et al. Preoperative controlling nutritional status (CONUT) score as a novel immunenutritional predictor of survival in non-metastatic clear cell renal cell carcinoma of $\leq 7 \mathrm{~cm}$ on preoperative imaging. J Cancer Res Clin Oncol 2019;145:957-65.

13. Kang HW, Seo SP, Kim WT, et al. Prognostic Impact of Nutritional Status Assessed by the Controlling Nutritional Status (CONUT) Score in Patients with Surgically Treated Renal Cell Carcinoma. Nutr Cancer 2018;70:886-94.

14. Borgmann $\mathrm{H}$, Musquera $\mathrm{M}$, Haferkamp A, et al. Prognostic significance of Fuhrman grade and age for cancer-specific and overall survival in patients with papillary renal cell carcinoma: results of an international multi-institutional study on 2189 patients. World J Urol 2017;35:1891-7.

15. Shalapour S, Karin M. Immunity, inflammation, and cancer: an eternal fight between good and evil. J Clin Invest 2015;125:3347-55.

16. Konigsbrugge O, Pabinger I, Ay C. Risk factors for venous thromboembolism in cancer: novel findings from the Vienna Cancer and Thrombosis Study (CATS). Thromb Res 2014;133 Suppl 2:S39-43.

17. Zhong H, Qian Y, Fang S, et al. Prognostic Value of Plasma Fibrinogen in Lung Cancer Patients: A MetaAnalysis. J Cancer 2018;9:3904-11.

18. Wang Y, Yin W, Wang Z, et al. Pretreatment plasma fibrinogen as an independent prognostic indicator of prostate cancer patients treated with androgen deprivation therapy. Prostate Cancer Prostatic Dis 2016;19:209-15.

19. Mei $Y$, Zhao S, Lu X, et al. Clinical and Prognostic Significance of Preoperative Plasma Fibrinogen Levels in Patients with Operable Breast Cancer. PLoS One 2016;11:e0146233.

20. Sun ZQ, Han XN, Wang HJ, et al. Prognostic significance of preoperative fibrinogen in patients with colon cancer. World J Gastroenterol 2014;20:8583-91.

21. Luo Y, Kim HS, Kim M, et al. Elevated plasma fibrinogen levels and prognosis of epithelial ovarian cancer: a cohort study and meta-analysis. J Gynecol Oncol 2017;28:e36.

22. Staton CA, Brown NJ, Lewis CE. The role of fibrinogen and related fragments in tumour angiogenesis and 
metastasis. Expert Opin Biol Ther 2003;3:1105-20.

23. Martino MM, Briquez PS, Ranga A, et al. Heparin-binding domain of fibrin(ogen) binds growth factors and promotes tissue repair when incorporated within a synthetic matrix. Proc Natl Acad Sci U S A 2013;110:4563-8.

24. Witsch E, Sela M, Yarden Y. Roles for growth factors in cancer progression. Physiology (Bethesda) 2010;25:85-101.

25. McMillan DC, Watson WS, O'Gorman P, et al. Albumin concentrations are primarily determined by the body cell mass and the systemic inflammatory response in cancer patients with weight loss. Nutr Cancer 2001;39:210-3.

26. Mikoshiba T, Ozawa H, Saito S, et al. Usefulness of Hematological Inflammatory Markers in Predicting Severe Side-effects from Induction Chemotherapy in Head and Neck Cancer Patients. Anticancer Res 2019;39:3059-65.

27. Espinosa E, Feliu J, Zamora P, et al. Serum albumin and other prognostic factors related to response and survival in patients with advanced non-small cell lung cancer. Lung Cancer 1995;12:67-76.

28. Ge LN, Wang F. Prognostic significance of preoperative serum albumin in epithelial ovarian cancer patients: a systematic review and dose-response meta-analysis of observational studies. Cancer Manag Res 2018;10:815-25.

29. Lien YC, Hsieh CC, Wu YC, et al. Preoperative serum albumin level is a prognostic indicator for adenocarcinoma of the gastric cardia. J Gastrointest Surg 2004;8:1041-8.

Cite this article as: Liu J, Gan Y, Song H, Zhu K, Zhang Q. The predictive value of the preoperative fibrinogen-albumin ratio on the postoperative prognosis of renal cell carcinoma. Transl Androl Urol 2020;9(3):1053-1061. doi:10.21037/tau-19873
30. Cho YK, Chung JW, Kim JK, et al. Comparison of 7 staging systems for patients with hepatocellular carcinoma undergoing transarterial chemoembolization. Cancer 2008;112:352-61.

31. Kwon WA, Kim S, Kim SH, et al. Pretreatment Prognostic Nutritional Index Is an Independent Predictor of Survival in Patients With Metastatic Renal Cell Carcinoma Treated With Targeted Therapy. Clin Genitourin Cancer 2017;15:100-11.

32. Hu K, Lou L, Ye J, et al. Prognostic role of the neutrophillymphocyte ratio in renal cell carcinoma: a meta-analysis. BMJ Open 2015;5:e006404.

33. Kim SI, Kim SJ, Kim SJ, et al. Diagnostic Test Accuracy of Glasgow Prognostic Score as a Prognostic Factor for Renal Cell Carcinoma: A Meta-Analysis. Am J Clin Oncol 2020;43:393-8.

34. Kim TW, Lee JH, Shim KH, et al. Prognostic significance of preoperative and follow-up neutrophil-to-lymphocyte ratio and platelet-to-lymphocyte ratio in patients with non-metastatic clear cell renal cell carcinoma. Investig Clin Urol 2019;60:14-20.

35. Tsujino T, Komura K, Hashimoto T, et al. C-reactive protein-albumin ratio as a prognostic factor in renal cell carcinoma - A data from multi-institutional study in Japan. Urol Oncol 2019;37:812.e1-812.e8. 